\title{
RAPIDLY INCREASING KERNELS
}

\section{HAROLD WIDOM}

In this note we determine the asymptotic form as $t \rightarrow \infty$ of the largest eigenvalue and corresponding eigenfunction of a positive symmetric kernel $k(x, y)$ on $(0, t)$ under the assumption that $k(x, y)$ increases regularly and rapidly as $x, y \rightarrow \infty$. Roughly speaking it is required that $k(x, y)$ grow more rapidly than every polynomial. The point is that $k(x, y)$ is in an appropriate sense close to $k(x, x)^{1 / 2} k(y, y)^{1 / 2}$ (see Lemma 3), a kernel with the single nonzero eigenvalue $\int_{0}^{t} k(x, x) d x$ and corresponding eigenfunction $k(x, x)^{1 / 2}$.

As corollaries we obtain the behavior of the largest eigenvalues and corresponding eigenfunctions of $k(x+y)$ on $(0, t)$ and $k(|x-y|)$ on $(-t, t)$ where $k$ is a rapidly increasing function of one variable.

Theorem. Assume $\gamma(x, y)=\log k(x, y)$ is real and symmetric, belongs to $C^{2}$, and satisfies

(i) $\gamma_{y} \geqq 0$,

(ii) $\lim _{x, y \rightarrow \infty} y \gamma_{y}(x, y)=\infty$,

(iii) $\gamma_{y y}=o\left(\gamma_{y}^{2}\right)$ and $\gamma_{y x}=o\left(\gamma_{y} \gamma_{x}\right)$ as $x, y \rightarrow \infty$.

Then the largest eigenvalue of $k(x, y)$ on $(0, t)$ is asymptotic to $k(t, t)^{2} / k^{\prime}(t, t)$ as $t \rightarrow \infty$. The eigenvalue is simple and its corresponding eigenfunction is asymptotic to $k(x, x)^{1 / 2}$. All other eigenvalues are $o\left(k(t, t)^{2} / k^{\prime}(t, t)\right)$.

Two points require explanation. We use the notation

$$
k^{\prime}(t, t)=\frac{d}{d t} k(t, t)
$$

The statement that the eigenfunction is asymptotic to $k(x, x)^{1 / 2}$ means that if the normalized eigenfunction be called $f$ and the function $k(x, x)^{1 / 2}$, normalized, be called $\phi$, then $\int_{0}^{t}|f(x)-\phi(x)|^{2} d x \rightarrow 0$ as $t \rightarrow \infty$.

Lemma 1. Let $g(x, y)$ satisfy the conditions (i)-(iii). Then as $x, t \rightarrow \infty$ we have

$$
\int_{0}^{t} e^{g(x, y)} d y \sim \frac{e^{g(x, t)}}{g_{y}(x, t)} .
$$

Proof. Let $M$ be so large that $g_{y}(x, y)>0$ for $x, y \geqq M$. Then

(1) $\int_{M}^{t} e^{g(x, y)} d y=\frac{e^{g(x, t)}}{g_{y}(x, t)}-\frac{e^{g(x, M)}}{g_{y}(x, M)}+\int_{M}^{t} \frac{g_{y y}(x, y)}{g_{y}(x, y)^{2}} e^{g(x, y)} d y$.

Received by the editors March 27, 1962. 
It follows from (iii) that if $x$ and $M$ are sufficiently large the integral on the right side of (1) has absolute value at most

$$
\epsilon \int_{M}^{t} e^{g(x, y)} d y .
$$

The rest of the right side of (1) is

$$
\frac{e^{g(x, t)}}{g_{y}(x, t)}\left[1-\frac{e^{g(x, M)}}{g_{y}(x, M)} / \frac{e^{g(x, t)}}{g_{y}(x, t)}\right] .
$$

The logarithm of the quotient inside the bracket is, by the mean value theorem,

$$
-(t-M)\left[g_{y}(x, M+\theta(t-M))-\frac{g_{y y}(x, M+\theta(t-M))}{g_{y}(x, M+\theta(t-M))}\right]
$$

for some $\theta$ in $(0,1)$. By (iii) this will be less than

$$
-\frac{1}{2}(t-M) g_{v}(x, M+\theta(t-M))
$$

if $x$ and $M$ are sufficiently large, and then by (ii) this will be less than $\log \epsilon$ if $t>2 M$. It follows that if $x$ and $M$ are sufficiently large and $t>2 M$ then

$$
\frac{1-\epsilon}{1+\epsilon} \leqq \int_{M}^{t} e^{g(x, y)} d y / \frac{e^{g(x, t)}}{g_{y}(x, t)} \leqq \frac{1}{1-\epsilon} .
$$

But for fixed $M$,

$$
\int_{0}^{M} e^{g(x, y)} d y=\frac{M}{t} \int_{0}^{t} e^{g\left(x, t^{-1} M y\right)} d y \leqq \frac{M}{t} \int_{0}^{t} e^{g(x, y)} d y
$$

by (i). Thus

$$
\int_{0}^{M}=o\left(\int_{0}^{t}\right)
$$

and the result follows.

LEMMA 2. Let $g(x, y)$ be symmetric and satisfy the conditions (i)-(iii). Then as $t \rightarrow \infty$ we have

$$
\int_{0}^{t} \int_{0}^{t} e^{o(x, y)} d y d x \sim \frac{4 e^{g(t, t)}}{g^{\prime}(t, t)^{2}}
$$

Proof. By Lemma 1 we can find an $M$ so that $x, t \geqq M$ imply

$$
\int_{0}^{t} e^{g(x, y)} d y \geqq(1-\epsilon) \frac{e^{g(x, t)}}{g_{y}(x, t)} .
$$


Then we have

$$
\begin{aligned}
\int_{0}^{t} d x \int_{0}^{t} e^{g(x, y)} d y \geqq(1-\epsilon) & \int_{M}^{t} \frac{e^{g(x, t)}}{g_{y}(x, t)} d x \\
=(1-\epsilon) & {\left[\frac{e^{g(t, t)}}{g_{x}(t, t) g_{y}(t, t)}-\frac{e^{g(M, t)}}{g_{x}(M, t) g_{y}(M, t)}\right.} \\
& \left.-\int_{M}^{t}\left(\frac{\partial}{\partial x} \frac{1}{g_{x}(x, t) g_{y}(x, t)}\right) e^{g(x, t)} d x\right] .
\end{aligned}
$$

It follows from (iii) and the symmetry of $g$ that if $M$ is sufficiently large the integral on the right side of (2) has absolute value at most $\epsilon \int_{M}^{t} e^{g(x, t)} / g_{y}(x, t) d x$. The logarithm of the quotient of the remaining two terms inside the bracket in (2) is, by the mean value theorem,

$$
\begin{gathered}
-(t-M)\left[g_{x}(M+\theta(t-M), t)-\frac{g_{x x}(M+\theta(t-M), t)}{g_{x}(M+\theta(t-M), t)}\right. \\
\left.-\frac{g_{y x}(M+\theta(t-M), t)}{g_{y}(M+\theta(t-M), t)}\right] .
\end{gathered}
$$

It follows from (ii) and (iii) and the symmetry of $g$ that this is less than $\log \epsilon$ if $M$ is sufficiently large and $t>2 M$. Then we have, since $g_{x}(t, t)=g_{y}(t, t)=\frac{1}{2} g^{\prime}(t, t)$,

$$
\int_{M}^{t} \int_{0}^{t} e^{g(x, y)} d y d x / \frac{4 e^{g(t, t)}}{g^{\prime}(t, t)^{2}} \geqq \frac{(1-\epsilon)^{2}}{1+\epsilon} .
$$

Therefore

$$
\liminf \int_{0}^{t} \int_{0}^{t} e^{g(x, y)} d y d x / \frac{4 e^{g(t, t)}}{g^{\prime}(t, t)^{2}} \geqq 1 .
$$

The proof of the opposite inequality with the lim sup is similar. To pass from the analogue of (3) to the analogue of (4) one uses the fact that for fixed $M$,

$$
\int_{0}^{M} \int_{0}^{t}=o\left(\int_{0}^{t} \int_{0}^{t}\right)
$$

as at the end of the proof of Lemma 1.

Lemma 3. As $t \rightarrow \infty$,

$$
\int_{0}^{t} \int_{0}^{t}\left[k(x, y)-k(x, x)^{1 / 2} k(y, y)^{1 / 2}\right]^{2} d y d x=o\left(\left(\int_{0}^{t} k(x, x) d x\right)^{2}\right) \text {. }
$$


Proof. If we expand the left side we see that it suffices to show

$$
\begin{aligned}
\int_{0}^{t} \int_{0}^{t} k(x, y)^{2} d y d x & \sim\left(\int_{0}^{t} k(x, x) d x\right)^{2}, \\
\int_{0}^{t} \int_{0}^{t} k(x, y) k(x, x)^{1 / 2} k(y, y)^{1 / 2} d y d x & \sim\left(\int_{0}^{t} k(x, x) d x\right)^{2} .
\end{aligned}
$$

But it follows from Lemma 1 with $g(x, y)=\gamma(y, y)$ that

$$
\int_{0}^{t} k(y, y) d y \sim \frac{e^{\gamma(t, t)}}{\gamma^{\prime}(t, t)},
$$

it follows from Lemma 2 with $g(x, y)=2 \gamma(x, y)$ that

$$
\int_{0}^{t} \int_{0}^{t} k(x, y)^{2} d y d x \sim \frac{e^{2 \gamma(t, t)}}{\gamma^{\prime}(t, t)^{2}}
$$

and it follows from Lemma 2 with $g(x, y)=\gamma(x, y)+\frac{1}{2} \gamma(x, x)+\frac{1}{2} \gamma(y, y)$ that

$$
\int_{0}^{t} \int_{0}^{t} k(x, y) k(x, x)^{1 / 2} k(y, y)^{1 / 2} d y d x \sim \frac{e^{2 \gamma(t, t)}}{\gamma^{\prime}(t, t)^{2}} .
$$

To deduce the theorem from Lemma 3 we need what is essentially a perturbation theorem, although none of those in the literature seems to apply directly. However the reader will recognize the following lemma as being the basis of one of the standard perturbation theorems.

LEMma 4. Let $A$ and $B$ be bounded self-adjoint operators with spectral families $E_{A}$ and $E_{B}$ respectively. Let $I$ be an interval of length $L, \Delta$ the distance from the end-points of $I$ to the spectrum of $B$, and $\delta=\|A-B\|$. Then if $\delta<\Delta$ we have

$$
\left\|E_{A}(I)-E_{B}(I)\right\| \leqq \frac{\delta L}{2 \Delta(\Delta-\delta)} .
$$

Proof. Let $C$ be the circle, described in the positive direction, with diameter $I$. Then

$$
\begin{aligned}
E_{A}(I)-E_{B}(I) & =\frac{1}{2 \pi i} \int_{C}\left[(A-\lambda)^{-1}-(B-\lambda)^{-1}\right] d \lambda \\
& =\frac{1}{2 \pi i} \int_{C}(A-\lambda)^{-1}(B-A)(B-\lambda)^{-1} d \lambda .
\end{aligned}
$$


and the integral is estimated in the obvious way using the given information.

We now prove the theorem. Let $A$ be the integral operator on $L_{2}(0, t)$ with kernel $k(x, y)$ and $B$ the operator with kernel $k(x, x)^{1 / 2} k(y, y)^{1 / 2}$. From (5),

$$
\|B\|=\int_{0}^{t} k(x, x) d x \sim \frac{k(t, t)^{2}}{k^{\prime}(t, t)} .
$$

It follows from Lemma 3 that $\|A-B\|=o(\|B\|)$ as $t \rightarrow \infty$. Let $I$ be the interval of length $\epsilon\|B\|$ with mid-point $\|B\|$. Since $\|B\| \rightarrow \infty$ it follows from Lemma 4 that

$$
\left\|E_{A}(I)-E_{B}(I)\right\| \rightarrow 0 .
$$

Since $E_{B}(I)$ is the projection onto the subspace spanned by $k(x, x)^{1 / 2}$ and since $\epsilon>0$ is arbitrarily small the first two statements of the theorem are established. Let $I^{\prime}$ be the interval $(\epsilon\|B\|,(1-\epsilon)\|B\|)$. Then $E_{B}\left(I^{\prime}\right)=0$ and it follows that for sufficiently large $t$ we have $E_{A}\left(I^{\prime}\right)=0$, so that every eigenvalue of $A$ other than the largest must be $\leqq \epsilon\|B\|$. Similarly every such eigenvalue must be $\geqq-\epsilon\|B\|$.

CoROllaRy 1. Let $k(x)=e^{\gamma(x)}, x \geqq 0$, where $\gamma$ is real, belongs to $C^{2}$, and satisfies

(i) $\gamma^{\prime} \geqq 0$,

(ii) $\lim _{x \rightarrow \infty} x \gamma^{\prime}(x)=\infty$,

(iii) $\gamma^{\prime \prime}(x)=o\left(\gamma^{\prime 2}\right)$ as $x \rightarrow \infty$.

Then the largest eigenvalue of $k(x+y)$ on $(0, t)$ is asymptotic to $k(2 t)^{2} / 2 k^{\prime}(2 t)$ as $t \rightarrow \infty$. The eigenvalue is simple and its corresponding eigenfunction is asymptotic to $k(2 x)^{1 / 2}$. All other eigenvalues are $o\left(k(2 t)^{2} / k^{\prime}(2 t)\right)$.

Corollary 2. Let $k$ be as in Corollary 1. Then the kernel $k(|x-y|)$ on $(-t, t)$ has eigenvalues asymptotic to $\pm k(2 t)^{2} / 2 k^{\prime}(2 t)$ as $t \rightarrow \infty$. These eigenvalues are simple and have corresponding eigenfunctions asymptotic to $k(2|x|)^{1 / 2}$ and $\operatorname{sgn} x k(2|x|)^{1 / 2}$ respectively. All other eigenvalues are $o\left(k(2 t)^{2} / k^{\prime}(2 t)\right)$.

Proof. Let $C$ be the integral operator on $L_{2}(0, t)$ with kernel $k(x+y), D$ the operator on $L_{2}(0, t)$ with kernel $k(|x-y|)$ and $U$ the mapping from $L_{2}(0, t)$ to $L_{2}(-t, 0)$ given by $U f(x)=f(-x)$. Then the operator on $L_{2}(-t, t)$ with kernel $k(|x-y|)$ is given in the usual way by the matrix

$$
\left[\begin{array}{cc}
D & C U^{*} \\
U C & U D U^{*}
\end{array}\right] \text {. }
$$


It follows from Corollary 1 that

$$
\left[\begin{array}{cc}
0 & C U^{*} \\
U C & 0
\end{array}\right]
$$

has the properties stated in Corollary 2. If we can show that $\|D\|$ $=o(\|C\|)$ we could then apply Lemma 4 , with $A$ given by (6) and $B$ given by (7). Now it is a consequence of Lemma 3 and (5) not only that $\|C\| \sim k(2 t)^{2} / 2 k^{\prime}(2 t)$ but also that $\|\mid C\| \sim k(2 t)^{2} / 2 k^{\prime}(2 t)$, where ||| || denotes Hilbert-Schmidt norm. Thus it suffices to show $\|D\|$ $=o(\|C\|)$. But

$$
\|||\|^{2}=\int_{0}^{t} \int_{0}^{t} k(|x-y|)^{2} d y d x=2 \int_{0}^{t} d x \int_{0}^{x} k(y)^{2} d y
$$

and

$$
\|C\|^{2}=\int_{0}^{t} \int_{0}^{t} k(x+y)^{2} d y d x=2 \int_{0}^{t} d x \int_{x}^{2 x} k(y)^{2} d y
$$

Since $\gamma(2 y)-\gamma(y)=y \gamma^{\prime}((1+\theta) y) \rightarrow \infty$ as $y \rightarrow \infty$ by (ii), we have $k(y)$ $=o(k(2 y))$ and so

$$
\int_{0}^{x} k(y)^{2} d y=o\left(\int_{x}^{2 x} k(y)^{2} d y\right)
$$

This proves $\||D|\|=o(\||C|\|)$.

Finally we remark upon the conditions we have imposed and growth conditions. In Corollary 1 , if $\gamma$ is nondecreasing and $\lim \gamma^{\prime \prime} / \gamma^{\prime 2}$ exists, finitely or infinitely, then it is easy to show that (ii) implies

$$
\frac{\gamma(x)}{\log x} \rightarrow \infty
$$

and that (ii) and (iii) are implied by

$$
\frac{\gamma(x)}{\log x} \uparrow \infty .
$$

Thus our requirement is roughly that $\gamma(x)$ increases more rapidly than $\log x$. This is the correct condition. For if $\gamma(x)=\alpha \log x$ with $\alpha$ a constant then $k(2 t)^{2} / 2 k^{\prime}(2 t)=\alpha^{-1} 2^{\alpha} t^{\alpha+1}$ while the Hilbert-Schmidt norm of $k(x+y)$ is $t^{\alpha+1}$ times a constant smaller than $\alpha^{-1} 2^{\alpha}$. 